\section{ORIGINAL RESEARCH}

\section{Arkadir}

R. Eichel

J.M. Gomori

T. Ben Hur

J.E. Cohen

R.R. Leker

\title{
Multimodal Reperfusion Therapy for Large Hemispheric Infarcts in Octogenarians: Is Good Outcome a Realistic Goal?
}

\begin{abstract}
BACKGROUND AND PURPOSE: MMRT may be beneficial in a subset of patients with large hemispheric stroke who cannot be treated with systemic thrombolysis. Because most previous studies only included relatively young patients, the outcome of very old patients given MMRT remains unknown.
\end{abstract}

MATERIALS AND METHODS: Consecutive patients with large hemispheric stroke treated with MMRT and admitted to intensive care were included. We compared neurologic and functional outcomes between patients younger and older than 80 years.

RESULTS: We included 14 patients older than 80 years and compared them with 66 patients who were younger than 80. Cerebrovascular risk factor profile, admission NIHSS scores, stroke etiology and pathogenesis, and procedure-related variables did not differ between the groups except for a higher prevalence of smoking in younger patients. Excellent target vessel recanalization (Thrombolysis in Myocardial Infarction score of 3 ) and good outcome at 90 days (modified Rankin Score $\leq 2$ ) were more common in younger patients $(45 \%$ versus $14 \%, P=.047$, and $41 \%$ versus $0 \%, P=.008$, respectively). In contrast, mortality rates were higher in octogenarians (43\% versus $17 \%$, respectively).

CONCLUSIONS: In this study, very old patients had higher chances of mortality and a very low probability of achieving functional independence even after MMRT. Further prospective studies are needed to examine the futility of MMRT in the very old.

ABBREVIATIONS: GP $\mathrm{IIb} / \mathrm{Illa}=$ glycoprotein IIb/IIla; IA = intra-arterial; MMRT = multimodal reperfusion therapy; TIMI = Thrombolysis in Myocardial Infarction; TOAST = Trial of ORG 10172 in Acute Stroke

L arge hemispheric ischemic stroke carries a mortality rate of close to $80 \%$, if left untreated. ${ }^{1,2}$ Most of these patients suffer from either internal carotid occlusions or proximal middle cerebral artery occlusions. Systemic thrombolysis is of limited benefit in these patients. ${ }^{3-6}$ In most cases, mortality is caused by brain herniation secondary to increased intracranial pressure resulting from stroke-associated edema. ${ }^{7-9}$ The only way to prevent such a malignant course of events seems to be rapid tissue reperfusion after arterial recanalization. The chances of recanalizing the occluded vessel and restoring perfusion appear to be somewhat higher with endovascular approaches that combine more than one treatment technique, ${ }^{10-20}$ and this is now considered a valid therapeutic approach in many patients with large strokes. Most previous series evaluating MMRT in large hemispheric strokes only included relatively younger patients, with 80 years being the usual upper age limit. ${ }^{21-24}$ However, the life expectancy in most Westernized countries is constantly increasing and clinicians will likely be faced with many more patients presenting with large stroke who are older than 80 years. Therefore, we

Received June 26, 2011; accepted after revision September 20.

From the Departments of Neurology (D.A., R.E., T.B.H., R.R.L.), Radiology (J.M.G., J.E.C.), and Neurosurgery (J.E.C), Hadassah-Hebrew University Medical Center, Jerusalem, Israel.

This study was supported by the Peritz and Chantal Scheinberg Cerebrovascular Research Fund and by the Sol Irwin Juni Trust Fund.

Please address correspondence to Dr. R.R. Leker, Department of Neurology, Hebrew University-Hadassah Medical Center, P.0. Box 12000, Jerusalem 91120 Israel; e-mail: leker@hadassah.org.il

EIndicates article with supplemental on-line tables.

http://dx.doi.org/10.3174/ajnr.A2916 aimed to evaluate whether octogenarian patients would benefit from MMRT if it was widely available.

\section{Materials and Methods}

We prospectively recruited consecutive patients presenting with large hemispheric stroke who underwent MMRT over the span of 5 years from 2005-2010 into our stroke registry, and the data were retrospectively analyzed. The institutional review board (Hadassah Medical Organization) authorized anonymous inclusion of patients into the consecutive data base without obtaining informed consent.

To be eligible for MMRT, patients had to have an initial NIHSS score of 15 and had to be previously independent $(m R S<2)$. Furthermore, all included patients had presented with carotid territory infarct within 8 hours of stroke onset. The diagnosis of internal carotid or proximal middle cerebral artery occlusion was established according to clinical findings that included hemiparesis/hemiplegia; sensory symptoms and evidence for cortical involvement, such as aphasia; neglect; or hemianopsia in various combinations. The diagnosis of large vessel occlusion had to be proved with CTA, MRA, or DSA in all patients. Most of the included patients underwent a full multiparametric stroke MR imaging protocol that included diffusion, perfusion, and susceptibility-weighted imaging as well as MR angiography and FLAIR. Exclusion criteria included evidence of large hemispheric infarction on the admission CT, defined as hypoattenuation covering more than one-third of the MCA territory, international normalized ration $>3$, and existing disease with limited life expectancy (eg, terminal cancer). Patients with small vessel disease were excluded, as were those presenting in deep coma and those with primary intracerebral or subarachnoid hemorrhage.

In this study, we compared patients who were younger than 80 years at the time of presentation with those older than 80 years. 
Clinical and demographic characteristics accrued included cerebrovascular risk profile, concomitant medications, time from symptom onset to initiation of endovascular procedure, and time from onset to reperfusion. Infarct etiology was classified, according to TOAST criteria, as cardioembolic, large artery atherothrombotic, other classified (eg, dissection), or unclassified. Lesion locations were classified as extracranial carotid, intracranial carotid, proximal MCA (M1), and distal MCA (M2).

All patients were admitted to the intensive care unit for at least 24 hours postprocedure. Neurologic deficits were determined with the NIHSS score, and functional deficits before admission and at 90 days postinfarct were evaluated with the mRS scale; good outcome was defined as an $\mathrm{mRS} \leq 2$.

Radiologic parameters were evaluated on entry CT/MR imaging and on the diagnostic and therapeutic angiography and follow-up CT/CTA. Flow was classified with the TIMI system (0, no flow; 1 , minimal flow; 2, residual stenosis; and 3, normal patent vessel).

The number and types of procedural modalities were also documented and studied in all patients. MMRT was defined as any combination of 3 or more therapeutic modalities from a list that included IA lytics, angioplasty, stent placement, IA GP IIb/IIIa antagonists, mechanical clot disruption (ie, repeated passage of the clot with the guidewire intended to mechanically break the clot) and application of clot retrieval devices. Of note, this was the standard of care at our facility and does not refer to patients with tortuous approach but rather to resistant clots.

Treatment complications including postprocedure hemorrhage and clinical deterioration without hemorrhage were also documented.

Statistical evaluations were performed with the Sigma-Stat package (SPSS, Chicago, Illinois). For univariate analysis patients with good outcome were compared with those with bad outcome using Student $t$ test or $\chi^{2}$ tests.

\section{Results}

Eighty consecutive patients fulfilling entry criteria were recruited into this preliminary study. Of those, 14 were older than 80 years and represented the study group (median age $82.5,28 \%$ men). They were compared with the reminder of the patients $(n=66)$ who were younger than 80 years at the time of presentation (median age $62.5,59 \%$ men). The baseline clinical and radiologic characteristics are presented in Online Table 1. All patients were independent before the procedure $(\mathrm{mRS} \leq 2)$. We estimate that approximately 30 other patients with large infarcts were eventually excluded from treatment based on our inclusion/exclusion criteria (mainly due to presentation later than 8 hours from symptom onset and significant existing disability before the current event), and these patients were not evaluated in the current study. Included octogenarians differed significantly from younger patients in that they smoked less often (0 versus 44\%, $P=$ $.005)$, but other baseline variables did not differ between the groups. Occluded segment distributions did not significantly differ between the groups (On-line Table 1). Of note, procedure-related variables-including type and site of vessel occlusion, onset to treatment time and time to vessel recanalization, number and types of procedural modalities used, and lesion length-did not differ between the groups (On-line Table 2). Good target vessel recanalization (TIMI 2-3) was achieved in 7 of the older patients (50\%) compared with 41 of the younger patients $(67 \%, P=.05)$. Excellent target vessel recanalization (TIMI 3) was achieved significantly more frequently in younger patients (45\% versus $14 \%, P=.047$ ).

At 7 days poststroke, 6 octogenarians (43\%) and 11 of the younger patients had died (17\%), but the difference between the groups was not significant $(P=.065)$.

The percentage of patients who achieved good outcome at 90 days, defined as a $\mathrm{mRS} \leq 2$ was significantly lower in the older-than- 80 group ( $0 \%$ versus $41 \%, P=.008)$. Two of our octogenarian patients reached a mRS of 3 at 90 days.

Seven of our patients (11\%) had hemorrhagic transformation of their infarcts. Three of these (4\%) were classified as symptomatic because they had confluent parenchymal hematoma that resulted in neurologic worsening. Of these, 2 were in the younger-than-80 group and 1 was in the octogenarian group.

Because most of our patients had cardioembolic events and because cardioembolism is related to poor outcome, we further compared patients with cardioembolic strokes in both groups. Patients with cardioembolic strokes who were younger than 80 differed from those that were over 80 in that they smoked more often $(46 \%$ versus $0 \% P=.002)$ and had lower NIHSS scores on day 1 and at discharge (13.4 \pm 7.1 versus $19.1 \pm 7.5, P=.016$, and $8.2 \pm 5.1$ versus $13.1 \pm 7.5$, $P=.01$, respectively). However, onset to treatment, NIHSS score on admission, and all other vascular risk factors, as well as treatment modalities used, did not differ between the groups. The chances of achieving an mRS of $\leq 2$ were significantly lower in octogenarians with cardioembolism ( $0 \%$ versus $46 \%, P=.002)$, and there was a trend toward lower mortality in the younger patients (17\% versus $54 \%, P=.059)$.

\section{Discussion}

The current preliminary study further expands the existing knowledge regarding the efficacy of MMRT in older patients with large, and often deadly, strokes. This is particularly important as the population in the Western world grows substantially older and many more octogenarians will likely present with large hemispheric strokes in the upcoming years, leading to the creation of a large burden on intensive care services.

Although we did not include a control group in this preliminary study, based on historical data, our results demonstrate that MMRT resulted in very high survival and good outcome rates in patients with severe strokes. However, the chances for good outcome were increased and the chances of mortality were reduced, especially in patients younger than 80 , and favorable outcomes were not observed in any of our octogenarian patients. Our results are in agreement with those of a pooled analysis of data from prospective trials and with those observed in a retrospective study. ${ }^{25,26}$ In both these reports, an age of over 80 years remained an important modulator of poor outcome after adjusting for recanalization status. It is important to note that, in our study, the percentage of patients with excellent vessel recanalization was significantly larger in younger patients, suggesting that the poor outcome observed in octogenarians could be related to failure of target vessel opening. Interestingly, in a recently published study, ${ }^{27}$ age was found to be an important modulator of futile recanalization and poor outcome after adjusting for recanalization state, sug- 
gesting that the impact of age on outcome may not solely depend on vessel recanalization.

Importantly, in the current study, octogenarian patients presented with similar stroke severity, etiology, and onset to treatment and onset to recanalization timeframes compared with younger patients. Admission to intensive care was a prerequisite of this study; therefore, admission settings are not responsible for the discrepant results between younger patients and octogenarians.

Furthermore, despite the very late intervention times in some of the patients, we could still achieve good outcomes with a very low complication rate. This suggests that, indeed, the therapeutic window of opportunity for reperfusion is much larger than previously thought. ${ }^{10,11}$ The low symptomatic intracerebral hemorrhage rates obtained in our group of patients is also reassuring, as such patients may still be good candidates for therapy.

Importantly, 2 of our elderly patients did reach a mRS of 3, which may still be looked upon as a reasonable outcome in patients with very large stroke, as these patients are still able to ambulate. Furthermore, chronologic age does not always equal biologic age, and therefore some octogenarians will probably still do well with treatment. Hence, we certainly cannot recommend withholding therapy in octogenarians, despite the high costs and the low chances for good functional outcome. However, our results and those of others ${ }^{25,26}$ suggest that prognostication should be more guarded in older patients.

Our study is limited by a relatively small number of included patients, and therefore we cannot exclude the possibility of missing statistical significance due to low power. Furthermore, approximately 30 other patients were excluded from the study because they had exclusion criteria that prevented their inclusion. Because we do not have full datasets for these patients, this may have introduced a bias in our results. Nevertheless, our results can be viewed as hypothesis-generating, and larger randomized studies exploring outcome after MMRT in younger versus older patients should be carried out in the future.

\section{Conclusions}

MMRT may be beneficial for most patients with large hemispheric strokes and significantly improves outcome especially in younger patients. Variables associated with increased chances of survival and good outcome, including successful recanalization, can only be determined during angiography. Therefore, our results suggest that efforts to recanalize the occluded artery may be considered in all patients with distal internal carotid or proximal middle artery occlusions, but that prognostication should be guarded in the very old patients with cardioembolic strokes.

\section{References}

1. Berrouschot J, Sterker M, Bettin S, et al. Mortality of space-occupying ("malignant") middle cerebral artery infarction under conservative intensive care. Intensive Care Med 1998;24:620-23
2. Hacke W, Schwab S, Horn M, et al. "Malignant" middle cerebral artery territory infarction: clinical course and prognostic signs. Arch Neurol 1996;53:309-15

3. Christou I, Felberg RA, Demchuk AM, et al. Intravenous tissue plasminogen activator and flow improvement in acute ischemic stroke patients with internal carotid artery occlusion. J Neuroimaging 2002;12:119-23

4. Derex L, Hermier M, Adeleine P, et al. Influence of the site of arterial occlusion on multiple baseline hemodynamic MRI parameters and post-thrombolytic recanalization in acute stroke. Neuroradiology 2004;46:883-87

5. Lee KY, Han SW, Kim SH, et al. Early recanalization after intravenous administration of recombinant tissue plasminogen activator as assessed by pre- and post-thrombolytic angiography in acute ischemic stroke patients. Stroke 2007;38:192-93

6. Linfante I, Llinas RH, Selim M, et al. Clinical and vascular outcome in internal carotid artery versus middle cerebral artery occlusions after intravenous tissue plasminogen activator. Stroke 2002;33:2066-71

7. Frank JI. Large hemispheric infarction, deterioration, and intracranial pressure. Neurology 1995;45:1286-90

8. Kasner SE, Demchuk AM, Berrouschot J, et al. Predictors of fatal brain edema in massive hemispheric ischemic stroke. Stroke 2001;32:2117-23

9. Qureshi AI, Suarez JI, Yahia AM, et al. Timing of neurologic deterioration in massive middle cerebral artery infarction: a multicenter review. Crit Care Med 2003;31:272-77

10. Abou-Chebl A, Bajzer CT, Krieger DW, et al. Multimodal therapy for the treatment of severe ischemic stroke combining GPIIb/IIIa antagonists and angioplasty after failure of thrombolysis. Stroke 2005;36:2286-88

11. Arnold M, Nedeltchev K, Mattle HP, et al. Intra-arterial thrombolysis in 24 consecutive patients with internal carotid artery T occlusions. J Neurol Neurosurg Psychiatry 2003;74:739-42

12. Flint AC, Duckwiler GR, Budzik RF, et al. Mechanical thrombectomy of intracranial internal carotid occlusion: pooled results of the MERCI and Multi MERCI Part I trials. Stroke 2007;38:1274-80

13. Ikushima I, Ohta H, Hirai T, et al. Balloon catheter disruption of middle cerebral artery thrombus in conjunction with thrombolysis for the treatment of acute middle cerebral artery embolism. AJNR Am J Neuroradiol 2007;28:513-17

14. Imai $\mathrm{K}$, Mori $\mathrm{T}$, Izumoto $\mathrm{H}$, et al. Clot removal therapy by aspiration and extraction for acute embolic carotid occlusion. AJNR Am J Neuroradiol 2006;27:1521-27

15. Imai $\mathrm{K}$, Mori $\mathrm{T}$, Izumoto $\mathrm{H}$, et al. Emergency carotid artery stent placement in patients with acute ischemic stroke. AJNR Am J Neuroradiol 2005;26:1249-58

16. Inoue $\mathrm{T}$, Kimura $\mathrm{K}$, Minematsu $\mathrm{K}$, et al. A case-control analysis of intra-arterial urokinase thrombolysis in acute cardioembolic stroke. Cerebrovasc Dis 2005;19:225-28

17. Jahan R, Duckwiler GR, Kidwell CS, et al. Intraarterial thrombolysis for treatment of acute stroke: experience in 26 patients with long-term follow-up. AJNR Am J Neuroradiol 1999;20:1291-99

18. Jovin TG, Gupta R, Uchino K, et al. Emergent stenting of extracranial internal carotid artery occlusion in acute stroke has a high revascularization rate. Stroke 2005;36:2426-30

19. Levy EI, Ecker RD, Horowitz MB, et al. Stent-assisted intracranial recanalization for acute stroke: early results. Neurosurgery 2006;58:458-63; discussion $458-63$

20. Zaidat OO, Suarez JI, Santillan C, et al. Response to intra-arterial and combined intravenous and intra-arterial thrombolytic therapy in patients with distal internal carotid artery occlusion. Stroke 2002;33:1821-26

21. Combined intravenous and intra-arterial recanalization for acute ischemic stroke: the Interventional Management of Stroke Study. Stroke 2004; 35:904-11

22. Alexandrov AV, Molina CA, Grotta JC, et al. Ultrasound-enhanced systemic thrombolysis for acute ischemic stroke. N Engl J Med 2004;351:2170-78

23. Smith WS, Sung G, Saver J, et al. Mechanical thrombectomy for acute ischemic stroke: final results of the Multi MERCI trial. Stroke 2008;39:1205-12

24. Khatri P, Hill MD, Palesch YY, et al. Methodology of the Interventional Management of Stroke III Trial. Int J Stroke 2008;3:130-37

25. Kim D, Ford GA, Kidwell CS, et al. Intra-arterial thrombolysis for acute stroke in patients $\mathbf{8 0}$ and older: a comparison of results in patients younger than $\mathbf{8 0}$ years. AJNR Am J Neuroradiol 2007;28:159-63

26. Qureshi AI, Suri MF, Georgiadis AL, et al. Intra-arterial recanalization techniques for patients 80 years or older with acute ischemic stroke: pooled analysis from 4 prospective studies. AJNR Am J Neuroradiol 2009;30:1184-89

27. Hussein HM, Georgiadis AL, Vazquez G, et al. Occurrence and predictors of futile recanalization following endovascular treatment among patients with acute ischemic stroke: a multicenter study. AJNR Am J Neuroradiol 31:454-58 